\title{
The Determination of Effects of Primary and Secondary Geometry of Tubular Linear Generator
}

\author{
Serdal Arslan ${ }^{1}$, Osman Gürdal ${ }^{2}$, Sibel Akkaya Oy ${ }^{3}$ \\ ${ }^{I}$ Birecik Vocational Higher School, Harran University, Birecik, Turkey \\ ${ }^{3}$ Faculty of Marine Sciences, Ordu University, Turkey
}

\begin{abstract}
In this paper, general information on the construction of tubular generators are given. Also, the numeric analyses were conducted by using $2 D$ and $3 D$ finite elements software. The output power, iron loss and cogging force change were compared by sensing the primary and secondary structural geometry of generator. Instead of the annular magnets, the magnets in the form of block were used mobile (secondary) part of generator. Also, analyses were conducted on the generator for two primary geometries.
\end{abstract}

Keywords: tubular linear generator, linear motor, generator design, electromagnetic design

\section{Introduction}

As known, the machines which transforms the mechanical energy into electric energy are named as the generator. It directly affects the topology of generator to be used whether the mechanical energy consists of circular or linear motion. As the linear induction generators have a variable speed force characteristic, they are used in the systems which produces electric from heat (instead of strling engine) and in Archimedes' wave swing system [1], [2]. As the vernier generator which is evaluated within the class of variable reluctance and the diagonal transverse have high force density, they become widespread in the wave energy applications (Archimedes Wave Swing) [3]. The permanent magnet synchronous generators may be adapted to almost all linear secondary systems in terms of speed and force characteristic and the power density. Although these creates a disadvantage in terms of that the performances of continuous magnets decrease at high temperature and the prices are high, they are preferred due to their high efficiencies. In this regards, the permanent magnet linear generator was preferred as the generator type. The tubular structure is preferred due to hat it has less stray flux and higher electromagnetic force (emf) compared to the flat structure [4,5]. They have some other advantages: there will be very low pressure on the linear bed due to that the high forces between the armatures (normal) are balanced (the sum of normal forces is equal to zero). Therefore, their life will be extended [6]. Other advantages are as follows: they have high compressive force and they show a perfect servo characteristic along with that the final effect at flat linear generators (final coil effector) is not seen, generally they are easily constructed, the requirement for using motion transformation mechanisms is eliminated, the mechanical losses are decreased and the reliability increases. It is desired that the forces of linear generators should not be asymmetric. Therefore, one-sided flat type is not preferred [7,8]. While the magnetic flux density at the air range of tubular generators have a stable value, the magnetic flux density changes at tetragon structure due to that the airway length is higher. Compared to the tubular generator, the iron and copper losses are much more at tetragon structure. Therefore, their efficiencies are lower [9]. Also, the tubular machines are classified depending on the flux direction (radial, axial, halbach). In this paper, the flux direction is determined as the neodymium magnets with radial flux. In this paper, the primary and secondary part of reciprocal collective coil wye-connected generator which had 6/4 hollow to pole ratio were changed. By using 2D and 3D finite elements software, the numeric analyses of tubular generator were conducted. By changing the primary and secondary structural geometry of generator, the change of output power, iron loss and cogging force were compared. Instead of the annular magnets, the block magnets were used at the mobile part (secondary) of generator. The analyses were conducted also for the two primary geometries of generator.

\section{Tubular Linear Generator Structures}

The magnets used at the permanent magnet tubular linear generators (PMTLG) are used as the neodymium magnets which have a high-energy density. For example; it is also required to take desired magnetic performance, operation temperature range, external demagnetized areas, weight, cost and positioning to the core into consideration. When the specifications of Neodymium-Iron-Boron magnets (energy density, magnetic flux density, etc.) are considered, they are commonly used in the generator applications. These magnets are demagnetized under the temperature and mechanical effects. Therefore, the magnets of which Cruie temperature is high (T>200) or UH-EH-VH/AH coded magnets are preferred. At PMTLGs, Samaloy 8SMC) core material and siliceous sheets are generally used. SMCs are the materials which have low starting permeability, low saturation induction and high loss compared to the laminated siliceous sheets. But it has lower losses at high 
frequencies compared to the siliceous sheets. Although the hysteresis losses are bad when it is compared with a single laminated siliceous sheet, their dynamic behaviors are better due to the loss of decreased eddy currents. Tansil300 (3.5\% SiFe lamination) showed a better performance compared to Somaloy 500 and Somaloy 700 materials in terms of force and power factor [10]. Also, the magnetic flux was equally distributed to all axes as SMCs had isotropic specifications [11]. The use of anisotropic amorp-fe alloy materials at tubular machines will change the dynamic effect of machine. The cost of a core material significantly depends on the magnetic characteristic. The core materials may be characterized as linear and nonlinear. The parameters which determine the specification of core material may be expressed as follows: magnetic permeability, saturation point, permanent magnetism, electrical resistance and coercivity. As known, the siliceous sheets are manufactured up to $0.65 \mathrm{~mm}$ and pressed at desired dimensions (by taking eddy loss into consideration). In this paper, M43 siliceous sheet was selected and analyses were conducted. In Table 1, the general specifications of this sheet were given in comparison with SMC.

Table 1. The Specifications belonging to M43-SMC materials

\begin{tabular}{|l|l|l|l|}
\hline GENERAL SPECIFICATIONS & $\begin{array}{l}\text { M43 Core Material } \\
\text { Specifications }\end{array}$ & $\begin{array}{l}\text { SMC (Somaloy 700) } \\
\text { Core Material Specifications }\end{array}$ & Units \\
\hline Alloy & $3 \%$ SiFe & Inorganic insulated iron particles & \\
\hline $\begin{array}{l}\text { Magnetic Flux Density at } \\
\mathrm{H}=10 \mathrm{KA} / \mathrm{m}\end{array}$ & 1.79 & 1.56 & $\mathrm{~T}$ \\
\hline Electrical Resistance & 0,42 & 400 & $\mu \Omega \mathrm{m}$ \\
\hline Density & 7,7 & 7,45 & $\mathrm{~g} / \mathrm{cm} 3$ \\
\hline Packing Factor & 0,96 & solid & $\mathrm{mm}$ \\
\hline Core Loss-50Hz & $3.57(1.5 \mathrm{~T}-50 \mathrm{~Hz})$ & $10(1 \mathrm{~T}-100 \mathrm{~Hz})$ & $\mathrm{W} / \mathrm{Kg}$ \\
\hline
\end{tabular}
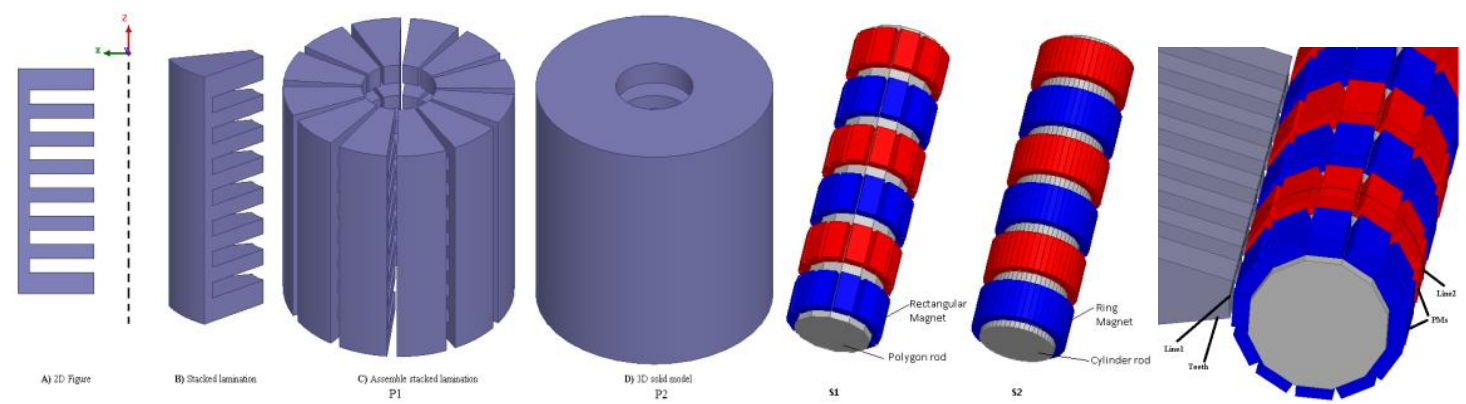

Figure 1. Different Primary and Secondary Images

Here, the basic primary image defined at 2D rz plane given with A are given. The image given with $\mathrm{B}$ is seen as the longitudinal sheet package is seen. It was accepted that the sheets were packed in the way that it will set 30-degree angle around $\mathrm{z}$ axis. By placing 12 units from sheet package, P1 primary given with $\mathrm{C}$ was obtained. P2 primary was created by cutting the siliceous sheets in the form of round and aligned along $\mathrm{z}$ axis. The secondary part was created in two ways. Steel 1020 material was defined in the form of dodecagon for placing block magnets. The neodymium magnets were placed on the material by taking the pole step and magnetization into consideration. This design was named as S1. Traditional tubular machine circular magnet structure and cylinder steel material are named as $\mathrm{S} 2$.

Table 2. Sizing Data

\begin{tabular}{|l|l|l|}
\hline Sizing Parameters & Dimension & Units \\
\hline Pole Step & 0.0275 & $\mathrm{~m}$ \\
\hline Hollow Step & 0.0183 & $\mathrm{~m}$ \\
\hline Primary external diameter & 0.1589 & $\mathrm{~m}$ \\
\hline Primary length & 0.1466 & $\mathrm{~m}$ \\
\hline Magnet length & 0.020 & $\mathrm{~m}$ \\
\hline Rod diameter & 0.038 & $\mathrm{~m}$ \\
\hline
\end{tabular}

\section{The Analysis of Finite Elements and Analysis Results}

Ansys-Maxwell; is one of the programs which are commonly preferred for the electromagnetic analyses that it shows approximate results given in the literature about that it has a wide drawing infrastructure, the solution options are redundant (The Transient State, Magnetostatic, Eddy currents Electrostatic, DC transmission, AC transmission), the analysis may be conducted by using finite elements methods, the machine model designed may be conducted with the auxiliary programs (Simplorer, Matlab, Ansys Workbench, etc.) which may perform the dynamic and analytical analysis. Therefore, the numerical analyses were conducted by 
using Ansys-Maxwell. As some geometries of primary and secondary core materials may not be transformed into 2D rz plane, 3D analysis was applied. With the aim of searching for the advantages and disadvantages that the geometries given in Figure 1 have provided against each other, the hollow height, back iron thickness, hollow width, internal diameter and external coil spirea number, magnet width and magnet thickness were taken equally.

Magnetostatic Analysis: If the motion of load in a closed circuit will be limited in the way that time-varying electric current will be stable. As known, the static magnetic analysis does not take the time-dependent effects such as eddy currents, transient state, etc. The density of magnetic flux is relevant to the determination of magnetic flux and energy stored in the magnetic field. In the static magnetic analysis, the magnetic models which both may saturate and may not saturate may be modelled. The FEM solvent may conduct 2D and 3D static magnetic analysis. The time-relevant parts of Maxwell Equations are accepted as zero. In the twodimensional analysis, the energy is calculated by calculating the vector analysis.

The magnetic flux entering a closed surface is equal to the flux leaving the closed surface.

$\nabla \cdot \overrightarrow{\mathrm{B}}=0$

A vector of which divergence is zero may be referred as the rotational of a vector unit:

$\overrightarrow{\mathrm{B}}=\nabla \times \overrightarrow{\mathrm{A}}$

Here, A is referred as the magnetic vector potential $(\mathrm{Wb} / \mathrm{m})$. By calculating the magnetic vector potential, the magnetic flux density is calculated. The equality between the magnetic field density and magnetic field intensity $(\mathrm{H})$ is given in (3). With this equation, the magnetic flux density is calculated.

$$
\mathrm{B}=\mu \mathrm{H}
$$

The current density in the areas where magnetic field intensity is fixed and stable, the current density is taken as zero. The point form of Amper's Law is given in (4).

$\nabla \times \vec{H}=\vec{J}$

The magnetic flux densities of primary and secondary combinations with magnetostatic analysis in given in Figure 2. When the figures are reviewed, it is seen that the magnets have not subjected to demagnetization. Also, the radial magnetic geometry provides much more flux transition in the core threads compared to block magnet. But the saturation of core material is out of question.

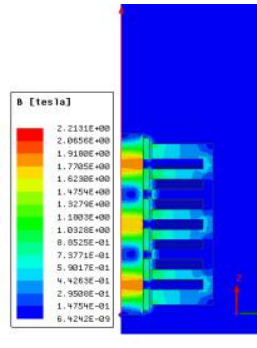

2D
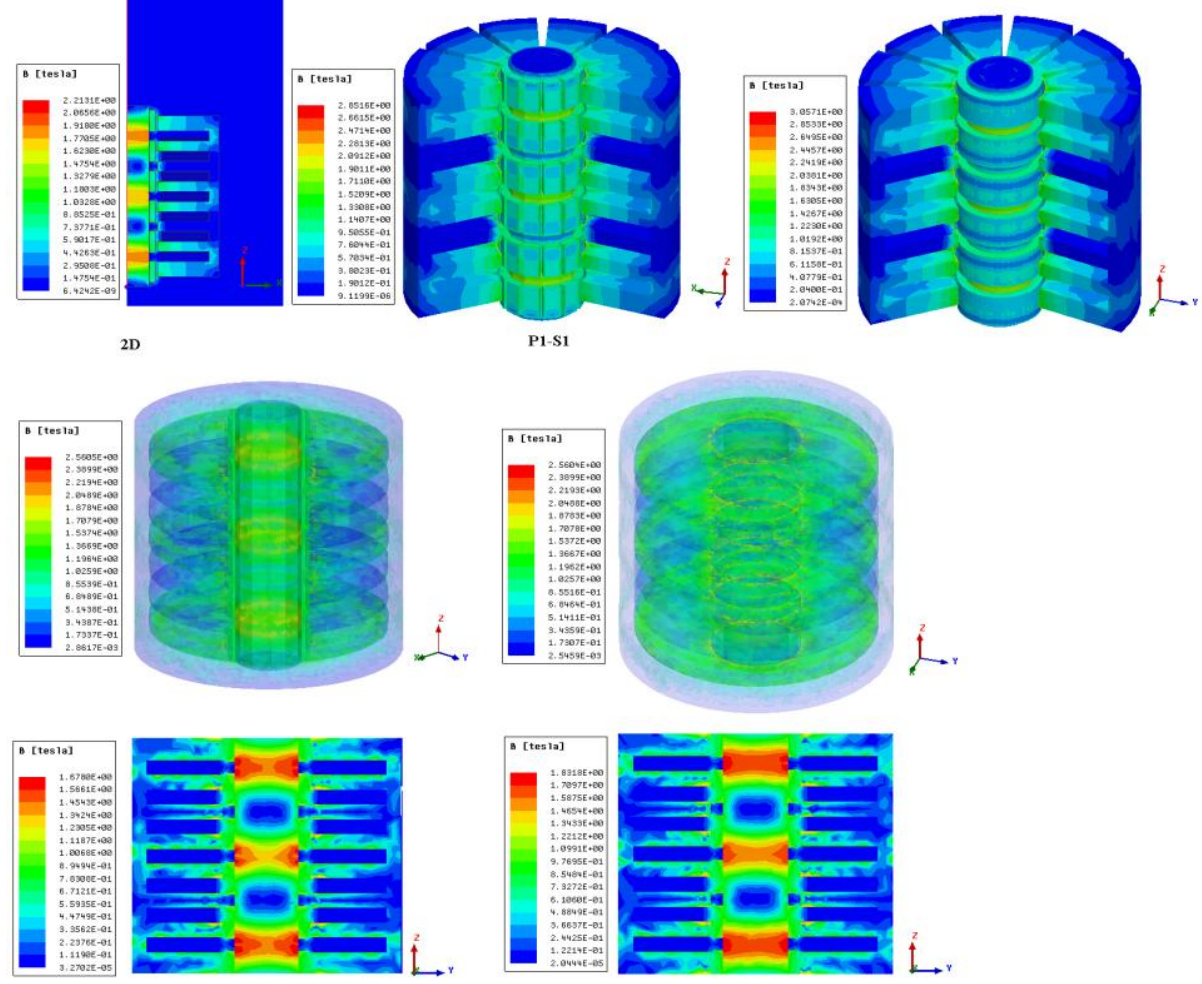

P2-S1

P2-s2

Figure 2. The magnetic flux density distribution of primary and secondary combinations 
In order to understand the structural differences, Line1 is defined along the primer as shown in Fig. In addition, Line 2 is defined as a half circle $\left(180^{\circ}\right)$ on the magnet (middle point of the air gap).

The flux density variation along these lines was investigated.
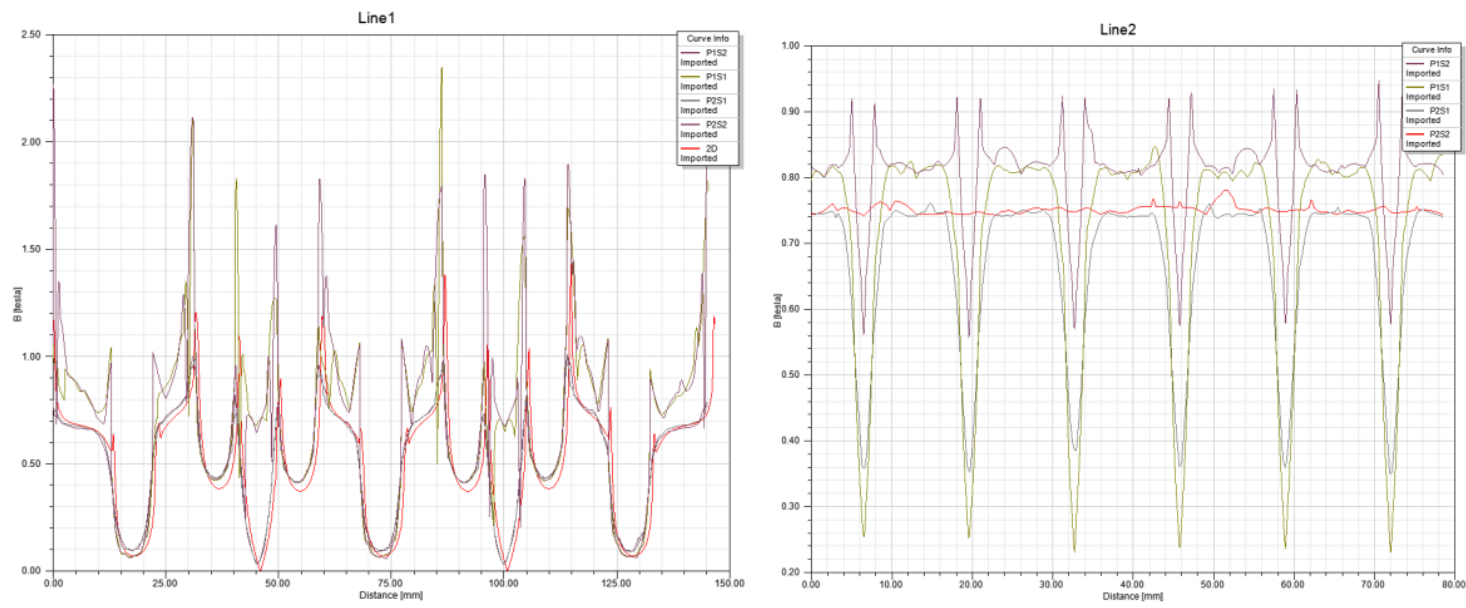

Figure 3. Fluctuation of flux density along Line 1 and Line 2

In the P2-S2 configuration, the flux density in the air gap remains constant. However, the magnetic flux density along the corner points of the poles is reduced in the segmented primary and block magnet secondary configurations. This is most likely the P1-S1 configuration.

Transient Analysis: Ansys-Maxwell 3D transient solvent uses the formulation of $\vec{T}-\Omega$. The Maxwell equations used in the transient analysis are as follows:

$$
\begin{aligned}
& \nabla \times \mathrm{H}=\mathrm{J} \\
& \nabla \times \mathrm{H}=\sigma \mathrm{E}
\end{aligned}
$$

Here, the correlation between the conductivity $(\sigma)$ and electric field $(\mathrm{E})$ indicates the current density $(\mathrm{J})$. The correlation between the current density and electric field is valid for the homogenous isotropic substances. The correlation between the current density and magnetic field intensity $(\mathrm{H})$ is due to the Ampere' Law. In the timevarying systems, the rotational of magnetic field intensity is referred as the change of magnetic flux density:

$\nabla \times \mathrm{H}=\frac{\partial \mathrm{B}}{\partial \mathrm{t}}$

The correlation between equation 5, 6 and 7 refers that the time-varying magnetic field will create the electrical field. The equation 8 may be indicated by using these two equations:

$$
\nabla \times \frac{1}{\sigma} \nabla \times \mathrm{H}+\frac{\partial \mathrm{B}}{\partial \mathrm{t}}=0
$$

The vector fields are represented with first order edge elements and the scalar fields are represented with the second order node unknowns. The current at the conductor defined as the surface may be defined in two ways: the spirea is defined for every phase coil. The first definition neglects the eddy effects. It allows for defining the coil parameters of generator (resistance, capacitance, inductance). Due to that the current is known at the time when the voltage is applied, the voltage drop for the surface defined current may be calculated for i. conductor node of current in (9) [12]:

$\mathrm{V}_{\mathrm{Ri}}=\iiint_{\mathrm{Rc}(\mathrm{i})} \mathrm{J}_{\mathrm{oi}}(\mathrm{E}+\mathrm{V} \times \mathrm{B}) \mathrm{dR}$

Here, J0i current density corresponds to the 1A net cycle i current and the external cycle $\mathrm{i}$ will disappear and $V_{R i}$ is given as the voltage drop. The induced e voltage may be calculated for every Rc(i) value:

$\underset{\mathrm{Rci}}{\mathrm{e}_{\mathrm{i}}}=-\iiint \mathrm{H}_{\mathrm{i}} \cdot \mathrm{BdR}$

Here, the integration is made on all conductor area. For the three-dimensional (3D) nonlinear transient state applications, the classical Newton-Raphson method is used [12]. In the transient analyses conducted, the operating speed and change of primary iron losses are given in Fig. 4: 

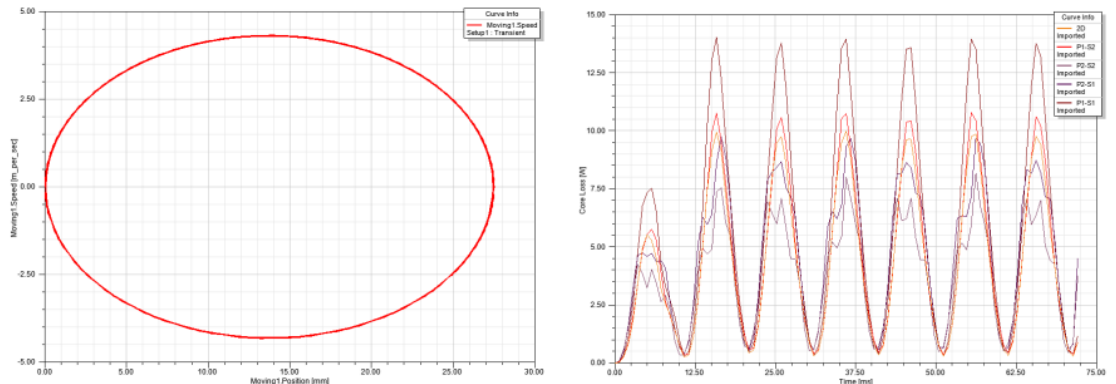

Figure 4. The speed-position change and the change of primary iron losses

No significant change was observed in the P1-S1 and P2-S1 configurations (the induced maximum stresses were 89.3 and 91.2, respectively), despite the variation of the flux density variation given in Fig. Because the effective magnetic flux density throughout the air gap is very close to each other. However, the maximum induced voltage in P1-S2 was 101.7V and the maximum induced voltage in P2-S2 was 93.4V.
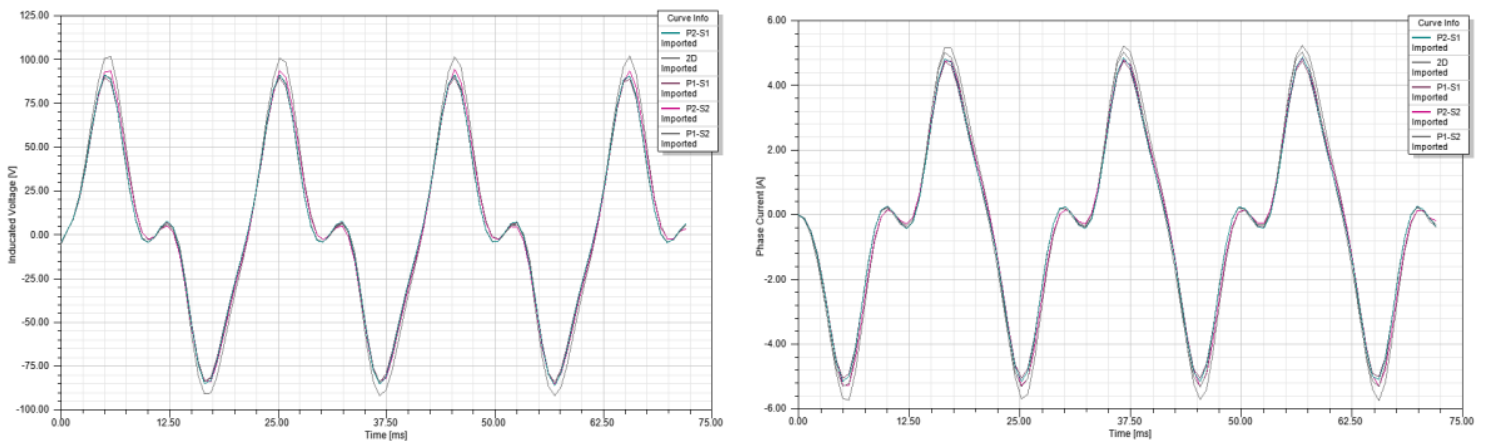

Figure 5. Induced voltage and phase current change according to combinations of primer and secondary

As the missing data of Secondary Steel 1020 material (W/kg) could not be obtained, only BH curve was defined. Therefore, the change of primary losses was only calculated. The cogging force and change of output power in accordance with the primary and secondary structures are given in Fig. 6 .
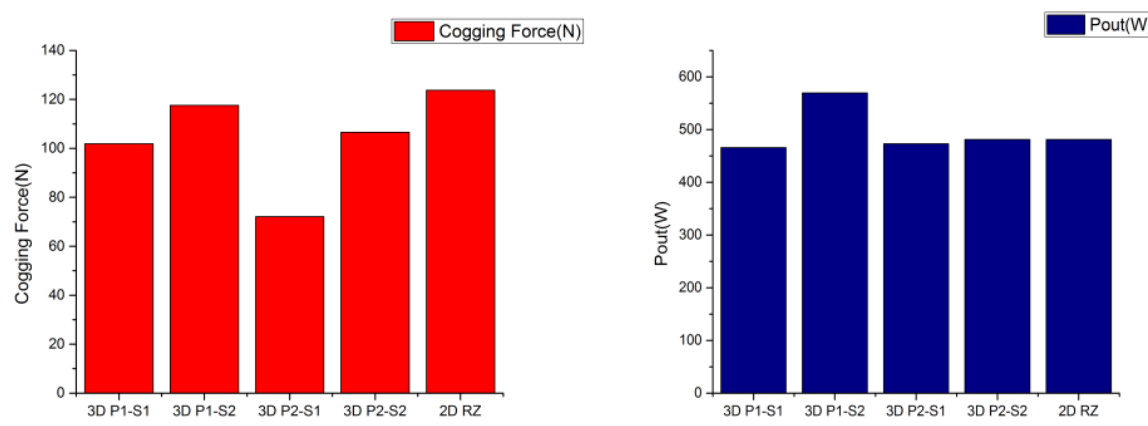

Figure 6. Cogging force, output power change

As known, it is desired that the cogging force as a result of attraction force between the primary core thread and secondary magnet shall be low without current at the coils. In order to decrease the cogging force, some techniques (hollow/pole combination [13], final thread width setting [14], hollow type [15], pole slip [16]) are applied. These methods significantly decrease the cogging force. In order to review the effect of primary and secondary structures, the methods were not applied in the study. The output power is $595 \mathrm{~W}$ for traditional P1-S2 and 481 W for P2-S2. Due to that the structure modelled at 2D rz had same geometric structure with P2$\mathrm{S} 2$, the output power was determined as approximately equal. The secondary structure created with block magnets led to a decrease on cogging force at the amount approximately $13 \%$ as well as that it did not significant differences in terms of output power. In this regards, using block magnets instead of ring magnets provides advantage. In Fig.7, the siliceous sheet and dodecagon secondary is given in order to create the generator geometry at the structure of P1-S1 and P2-S2. 

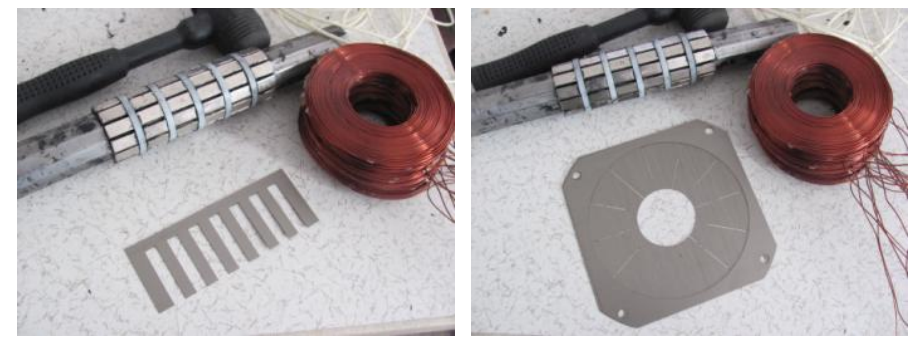

Figure 7. The image of P1-S1 and P2-S1 generator parts, respectively for prototype production

\section{Conclusion}

In this paper, the effects of tubular alternator primary and secondary geometry on the output power, cogging force and iron losses were reviewed. As distinctive from the traditional geometry, the use of block magnets at secondary part will not change the output power. But it decreases the demagnetization of magnets as well as that it leads to a decrease on the cogging force. The generator prototype production will be provided by combining the secondary and traditional primary structures with block magnet which will be produced in the future studies. The no-load and loaded experimental tests will be conducted.

\section{References}

[1] L. Wenlong, Design, analysis and application of low-speed permanent magnet linear machines, Unpublished Phd Thesis, The University of Hong Kong, Department of Electrical and Electronic Engineering, Hong Kong, 2012.

[2] L. Dall'Ora, Analysis and Design of a Linear Tubular Electric Machine for Free-piston Stirling Micro-cogeneration Systems, Unpublished Phd. Thesis, University of Padova, Italy, 2014.

[3] W. Li and K.T. Chau, K., Simulation of a linear permanent magnet vernier machine for direct-drive wave power generation. In International Conference on Electrical Machines and Systems (ICEMS) Proceedings, 2011, 1-6.

[4] H. Arof, A.M. Eid and K.M. Nor, Permanent magnet linear generator design using finite element method. In Electrical, Electronic and Computer Engineering, ICEEC'04 International Conference on, 2004, 893-896.

[5] S.R. Huang, H.T. Chen, C.H. Chung, C.Y. Chu, G.C. Lia and C.C. Wub, Multivariable direct-drive linear generators for wave energy, Applied Energy, 100, 2012, 112-117.

[6] B. Schmülling, M. Leßmann, B. Riemer and K. Hameyer, K. The multi-slice method for the design of a tubular linear motor with a skewed reaction rail. COMPEL-The international journal for computation and mathematics in electrical and electronic engineering, 29(5), 2010, 1184-1194.

[7] A. Chen, W.M. Arshad, P. Thelin and P. Zheng, P., Analysis and optimization of a longitudinal flux linear actuator for hybrid electric vehicle applications. IEEE Symp. Vehicle Power and Propulsion, 2004.

[8] J. Ribeiro and I. Martins, Development of a low speed linear generator for use in a wave energy converter. International Conference on Renewable Energies and Power Quality ICREPQ'10, Spain, 2010.

[9] C.A. Oprea, C.S. Martis, F.N. Jurca, D. Fodorean and L. Szabo, L., Permanent magnet linear generator for renewable energy applications: Tubular vs. four-sided structures, International Conference on in Clean Electrical Power (ICCEP), $2011,588-592$.

[10] J. Wang and D. Howe, Influence of soft magnetic materials on the design and performance of tubular permanent magnet machines, IEEE Transactions on Magnetics, 41(10), 2005, 4057-4059.

[11] F. Milanesi, F., Design optimization and control strategies for PM Multiphase Tubular Linear Actuators, Unpublished Master Thesis, Department of Electrical Engineering Faculty of Engineering University of Bologna, Italy, 2009.

[12] Ansoft Corporation, "Maxwell 3D technical notes", 200-1045 (2002).

[13] L. Wenlong, L., Design, analysis and application of low-speed permanent magnet linear machines. Unpublished Phd Thesis, Department of Electrical and Electronic Engineering, The University of Hong Kong, 2012.

[14] A.L. Li, B.C. Zhang and C.B. Kou, C. B., Analysis and Suppression of Detent Force in Tubular Linear Electromagnetic Launcher for Space Use, In Electromagnetic Launch Technology, 14th Symposium on, 2008,1-4.

[15] Y.W. Zhu and Y.H. Cho, Detent Force Reduction in Permanent-Magnet Linear Synchronous Motor Utilizing Auxiliary Poles, ELECTROMOTION 2009 - EPE Chapter 'Electric Drives' Joint Symposium, Lille, France, 1-3 July 2009.

[16] N. Bianchi, S. Bolognani and A.D.F. Cappello, Reduction of cogging force in PM linear motors by pole-shifting. IEEE Proceedings-Electric Power Applications, 152(3), 2005, 703-709. 